\title{
Comparison of 4- and 64-slice CT scanning in the diagnosis of pulmonary embolism
}

Renée A. Douma'; Herman M. A. Hofstee²; Cornelia Schaefer-Prokop33; Jan Hein T. M. van Waesberghe4; Rutger J. Lely4; Pieter W. Kamphuisen"; Victor E. A. Gerdes ${ }^{1,5}$; Mark H. H. Kramer2; Harry R. Büller ${ }^{1}$

${ }^{1}$ Department of Vascular Medicine, Academic Medical Center, Amsterdam, the Netherlands; ${ }^{2}$ Departments of Internal Medicine, VU University Medical Center, Amsterdam, the Netherlands; ${ }^{3}$ Department of Radiology, Academic Medical Center, Amsterdam, the Netherlands; ${ }^{4}$ Department of Radiology, VU University Medical Center, Amsterdam, the Netherlands, and ${ }^{5}$ Department of Internal Medicine, Slotervaart Hospital, Amsterdam, the Netherlands

\begin{abstract}
Summary
With the introduction of multi-detector row $\mathrm{CT}(\mathrm{MDCT})$, sensitivity to diagnose pulmonary embolism (PE) has greatly improved. The use of newer generation CT-scans may lead to a higher prevalence and a different distribution of PE. We compared 64-slice with 4-slice MDCT regarding prevalence and distribution of $\mathrm{PE}$, the number of inconclusive test results and inter-reader variability. CT-scans from a random sample of 110 consecutive patients who underwent 4-slice CT-scanning were compared with 64-slice CT-scans from 107 patients from a second cohort. Three radiologists independently reassessed all CT-scans. Consensus was reached in case of disagreement between the readers. Final diagnosis of PE was categorised as central, segmental or subsegmental by the thrombus' most proximal end. The prevalence of PE was $24 \%$ (26/110, 95\% confidence interval $[\mathrm{Cl}] 17-32 \%)$ and 22\% (24/107, $16-31 \%)$ for the 4 -slice and 64 -slice cohort, respectively. The preva-
\end{abstract}

Correspondence to:

Renée Douma, MD

Department of Vascular Medicine

Academic Medical Center

Meibergdreef 9, Suite F4-143

1105 AZ Amsterdam, the Netherlands

Tel.: +31 205668274 , Fax: +31 206968833

E-mail: r.a.douma@amc.uva.nl lence of isolated subsegmental emboli was $2 / 26(7.7 \% ; 2.1-24 \%)$ and $5 / 24(21 \% ; 9.2-41 \%)$, respectively $(p=0.424)$. The number of inconclusive scans was $10 \%$ in both cohorts, mostly due to movement artefacts and suboptimal intravascular contrast, respectively. The inter-reader agreement between the three readers was 0.70 for the 4-slice scans and 0.68 for the 64 -slice scans. Although absolute prevalence of PE was equal in both cohorts, there was a trend towards more subsegmental PE with 64-slice CT. In a multi-reader setting, the number of inconclusive examinations was higher than quoted for clinical management studies, indicating that the diagnosis of PE with MDCT could be less straightforward than assumed.

\section{Keywords}

Pulmonary embolism, venous thromboembolism, multi-detector row CT scan, diagnosis

Received: June 26, 2009

Accepted after major revision: September 7, 2009

Prepublished online: October 26, 2009

doi:10.1160/TH09-06-0406

Thromb Haemost 2010; 103: 242-246

\section{Introduction}

Computed tomographic pulmonary angiography (CTPA) is nowadays the accepted state-of-the art test in the diagnostic workup of patients with suspected pulmonary embolism (PE). Initially, critics were sceptical about the accuracy of detecting small emboli in segmental and subsegmental arteries with early single-slice detector computed tomography (CT) (1). However, with the introduction of multi-detector row CT (MDCT), visualisation of small peripheral arteries has greatly improved yielding an increased sensitivity for the detection of small subsegmental emboli (2-4). With the advent of newer generations detector row CT scans (16-/64-slice MDCT, up to even 256-slice MDCT or higher more recently) it is conceivable that the number of patients in whom only isolated small peripheral emboli are seen (i.e. involvement of subsegmental arteries, without involvement of the more central arteries) increases, with several unknown consequences.
We therefore wanted to compare 'low'-multi-detector row CT scans (i.e. 4-slice) with more modern 'high-end'-multi-detector row CT scans (i.e. 64-slice) for differences in the PE detection pattern. Unfortunately, a direct comparison between older and newer generation MDCT scanners in the same patients would require two scans per patient, which is unethical due to the radiation exposure. Therefore, we used two sets of patients to compare 4-slice and 64-slice MDCT, with re-evaluation of the CT scans by three radiologists. We expected an absolute increase in PE prevalence in the 64-slice cohort, with a higher proportion of isolated subsegmental pulmonary emboli (ISSPE) compared with the 4-slice cohort. We also expected to find less inconclusive test results with a 64-slice scanner, due to a higher diagnostic accuracy. Finally, we investigated the inter-reader variability to detect PE. 


\section{Methods}

\section{Patients}

This study was performed by evaluating MDCT scans from two cohorts of patients with suspected PE. Patients with a 'likely' clinical probability according to the Wells rule (5) or an abnormal D-dimer test result (Tinaquant ${ }^{\circledR}$, Roche Diagnostica, Mannheim, Germany, cut-off $\leq 500 \mu \mathrm{g} / \mathrm{l})$, and in whom a 4 -slice CTPA or 64-slice CTPA had been performed, were included. The first cohort consisted of a randomly selected sample of consecutive patients included in a large prospective diagnostic management study at the Academic Medical Center, Amsterdam, the Netherlands, between November 2002 and August 2004 (6). This study evaluated the clinical effectiveness of a simplified algorithm using the dichotomised Wells rule, D-dimer testing and CT in patients with suspected PE. The institutional review board approved the study protocol. In- and exclusion criteria have been published previously (6). At admission, the clinical probability was calculated by the treating physician using the Wells score. Among patients with a likely clinical probability (Wells score $>4$ ) or an abnormal D-dimer test PE was confirmed or excluded with CT scanning. For this analysis, a random sample of patients who underwent 4-slice CT scanning was selected using SPSS, to match the number of patients included in the second cohort. Besides the sample size, there were no requirements or restrictions in selecting the patients.

The second cohort consisted of consecutive in- and outpatients with suspected acute PE at the VU University Medical Center, also in Amsterdam, the Netherlands, between January and June 2006. The patients underwent the same diagnostic algorithm as in the first cohort and the patient referral pattern was the same for both hospitals. Patients with a likely clinical probability for PE (Wells $>4$ ) or an abnormal D-dimer test, and in whom a 64-slice CT was made, were included. The protocol had been approved by the institutional review board.

\section{MDCT scanning protocol}

CT pulmonary angiography scans were performed using 4-slice (first cohort) and 64-slice (second cohort) MDCT scanners. The scans had been made using state-of-the art protocols.

In the first cohort, according to the study protocol of the Christopher study (6), images were acquired with a 4-row MDCT scanner with $4 \times 1 \mathrm{~mm}$ slice collimation and a pitch of 1.5. Images for evaluation were reconstructed with $1.25 \mathrm{~mm}$ width and $1.2 \mathrm{~mm}$ increment. Using automatic bolus tracking (main pulmonary artery), $80-100 \mathrm{ml}$ contrast medium $(350 \mathrm{mg}$ iodium $/ 100 \mathrm{ml}$ ) was injected intravenously with a flow of $4 \mathrm{ml} / \mathrm{seconds}$.

In the second cohort, images were acquired with a $2 \times 32$ detector row MDCT scanner with $24 \times 1.2 \mathrm{~mm}$ slice collimation and a pitch of 0.75 . Images for evaluation were reconstructed with 1.5 $\mathrm{mm}$ width and $1 \mathrm{~mm}$ increment. Also using automatic bolus tracking (main pulmonary artery), $70 \mathrm{ml}$ contrast medium (30 mg io- dium/100ml) was injected intravenously with a flow of $4 \mathrm{ml} / \mathrm{sec}$ onds.

All images were acquired in the cranio-caudal direction with the z-axis coverage and the fields of view chosen to include the entire thorax, from the apex to the base of the lungs. Patients were examined in single breath-hold technique.

\section{MDCT assessment}

Three radiologists independently reassessed the CT scans. They came from both institutions, all were board certified and had experience of reading PE-CT scans for more than seven years. The mages were read independently by the three observers in different random order. All images were evaluated under identical reading conditions in one of the two institutions using the same reading station. Due to differences in image quality and in the design of the image display (due to the different scanner types), the readers were not completely blinded to the type of MDCT. In case of disagreement, a consensus reading was carried out to come to an agreement. CT scans were scored according to predefined criteria, which were assessed in a pilot reading session. The readers were unaware of any clinical information. The pulmonary arteries were evaluated up to and including the subsegmental vessels from the level of the aortic arch to the lowest hemidiaphragm. PE was diagnosed if contrast material outlined an intraluminal defect or if a vessel was totally occluded by low-attenuation material on at least two adjacent slices. Per finding, the location of the thrombus was recorded by its most proximal end. Standard nomenclature, derived from Boyden (7) and from Jackson and Huber (8) was used to identify the segmental and subsegmental structures. Final diagnosis of PE was categorised as central, segmental or subsegmental by the thrombus' most proximal end. The images were determined as inconclusive if no definite consensus could be reached by the three observers due to patient related factors, technical reasons such as poor opacification, artefacts (i.e. pulsation, breathing or flow artefacts) or due to the presence of ambiguous image findings (when it was simply too difficult to discriminate between thrombus or no thrombus, for no other reason than the finding itself).

\section{Statistical analysis}

Clinical characteristics of study patients from the two cohorts were compared using a chi ${ }^{2}$ test or Fisher's exact test for qualitative variables, and a Student t-test for continuous variables. Prevalence of $\mathrm{PE}$ and distribution were calculated along with their 95\% confidence intervals $(\mathrm{CI})$. To express inter-reader agreement the multi-reader kappa $(\kappa)$ coefficient was calculated. All analyses were performed using SPSS software, version 15.0, SPSS, Inc., Chicago, and Confidence Interval Analysis (CIA, version 1.0; Gardner $\mathrm{MJ})$. 


\section{Results}

In the 64-slice cohort, 117 consecutive patients were included. Of these patients, five were excluded from analysis because of incomplete data storage (only the $5 \mathrm{~mm}$ thick reconstructed sections

Table 1: Baseline characteristics of the study population.

\begin{tabular}{|c|c|c|}
\hline & $\begin{array}{l}\text { 4-slice }(n=110) \\
n(\%)\end{array}$ & $\begin{array}{l}\text { 64-slice }(n=107) \\
n(\%)\end{array}$ \\
\hline Age in years, mean (SD) & $57(19)$ & $59(19)$ \\
\hline Female gender, n (\%) & $64(58)$ & $65(61)$ \\
\hline Outpatients, n (\%) & $68(62)$ & $75(70)$ \\
\hline $\begin{array}{l}\text { Duration of complaints, median } \\
\text { (IQR) }\end{array}$ & $2(1-6)$ & $2(1-4)$ \\
\hline Personal history of VTE, $\mathrm{n}(\%)$ & $10(9)$ & $8(8)$ \\
\hline Active malignancy, n (\%) & $28(26)$ & $20(20)$ \\
\hline COPD, n (\%) & $13(12)$ & $11(11)$ \\
\hline Heart failure, $n(\%)$ & $15(14)$ & $8(8)$ \\
\hline Estrogen suppletion, $\mathrm{n}(\%)^{1}$ & $9(8)$ & $9(9)$ \\
\hline Wells rule more than $4, \mathrm{n}(\%)$ & $65(60)$ & $65(66)$ \\
\hline
\end{tabular}

$S D$, standard deviation ; IQR, inter-quartile range ; VTE, venous thromboembolism ; COPD, chronic obstructive pulmonary disease; ${ }^{1}$ of females only.

Table 2: Anatomical distribution of pulmonary emboli (PE) visualised by 4-slice and 64-slice multidetector-row computed tomography, according to the most proximal level of the pulmonary arterial tree.

\begin{tabular}{|c|c|c|c|}
\hline & $\begin{array}{l}\text { 4-slice MDCT } \\
\%(95 \% \mathrm{Cl})\end{array}$ & $\begin{array}{l}\text { 64-slice MDCT } \\
\%(95 \% \mathrm{Cl})\end{array}$ & P-value \\
\hline Central PE & $62(43-78)$ & $50(31-69)$ & 0.412 \\
\hline Segmental PE ${ }^{1}$ & $31(17-50)$ & $29(15-49)$ & 0.902 \\
\hline Subsegmental PE² & $\begin{array}{l}7.7(2.1-24) \\
n=2 / 26\end{array}$ & $\begin{array}{l}21(9.2-41) \\
n=5 / 24\end{array}$ & 0.424 \\
\hline
\end{tabular}

MDCT, multidetector-row computed tomography; $\mathrm{Cl}$, confidence interval; $\mathrm{PE}$, pulmonary embolism; ${ }^{1}$ weighted average from three readers; ${ }^{2}$ by consensus reading.

Table 3: Inconclusive CT results ${ }^{1}$.

\begin{tabular}{|c|c|c|c|}
\hline \multicolumn{2}{|c|}{ Inconclusive CT results ( $\mathrm{n}$ ) } & \multirow{2}{*}{$\begin{array}{l}\text { 4-slice CT } \\
\text { n }(\%, 95 \% C I) \\
33 / 330 \\
\end{array}$} & \multirow{2}{*}{\begin{tabular}{|l|l} 
64-slice CT \\
n (\%, 95\%Cl) \\
$30 / 321$ \\
\end{tabular}} \\
\hline \multirow{4}{*}{ Due to: } & & & \\
\hline & Artefacts $^{2}$ & $12(36,22-53)$ & $3(10,3.5-26)$ \\
\hline & Timing $^{3}$ & $9(27,15-44)$ & $18(60,42-75)$ \\
\hline & Dubious findings ${ }^{4}$ & $12(36,22-53)$ & $9(30,17-48)$ \\
\hline \multicolumn{4}{|c|}{$\begin{array}{l}{ }^{1} \text { Pooled results from the three readers. }{ }^{2} \text { Artefacts: pulsation, breathing or flow } \\
\text { artefacts. }{ }^{3} \text { Timing: poor opacification, poor timing of contrast material in the } \\
\text { pulmonary arteries and the moment of scanning. }{ }^{4} \text { Dubious findings: the finding } \\
\text { in itself was difficult to interpret, in the absence of other reasons which might } \\
\text { complicate the interpretation. }\end{array}$} \\
\hline
\end{tabular}

were available for retrospective analysis) and in another five patients the CDROM was missing in the moment of re-evaluation. These 10 patients were excluded from the analysis, leaving $107 \mathrm{pa}-$ tients for the current analysis. From the 378 patients analysed with 4-slice CT, a random sample of 110 was selected. Baseline characteristics of the two patient groups are shown in Table 1. Both groups were comparable with respect to age, gender, duration of complaints, risk factors for VTE and the prevalence of 'likely' clinical probability.

\section{Prevalence and distribution of PE}

The overall prevalence of PE by consensus was 24\% (26/110; 95\% CI $17-32 \%)$ and $22 \%(24 / 107 ; 95 \%$ CI 16-31\%) for the 4-slice and 64-slice cohort, respectively. The distribution of emboli is depicted in Table 2. Subsegmental emboli as the most proximal manifestation of PE were present in two of the 26 patients with PE in the 4-slice cohort $(7.7 \%$; 95\% CI, 2.1-24\%), whereas this figure was five of 24 patients $(21 \%$; 95\% CI, 9.2-41\%) in the 64-slice cohort. This difference did not reach significance $(\mathrm{p}=0.424)$.

\section{Inconclusive results and interreader agreement}

Scans were marked as inconclusive by consensus in $10 \%$ in both cohorts: 11 scans in the 4-slice cohort (10\%, 95\% CI 5.7-17\%) and 11 scans in the 64 -slice cohort $(10 \%, 95 \%$ CI $5.8-18 \%)$, respectively. The reason for an inconclusive outcome was marked by each reader individually and is depicted in Table 3. Among the 4-slice scans, inconclusive results were most often due to artefacts (pooled results, $n=12$ ), while with 64-slice scans, this was most often caused by suboptimal intravascular contrast (pooled results, $n=18$ ).

The inter-reader agreement between the three readers was comparable for the two groups: the $\kappa$-value for the three observers was 0.70 for the 4 -slice CT scan and 0.68 for the 64 -slice scan (range of kappa between two readers 0.62 to 0.80 and 0.64 to 0.71 for the 4 and 64-slice cohorts, respectively). None of the readers performed significantly better or worse compared to the other readers (data not shown).

\section{Discussion}

The two main findings of this indirect comparison between 4- and 64-slice CT scans in patients with suspected PE were a trend towards a higher prevalence of subsegmental emboli with the 64-slice CT and secondly a relatively high number of scans rated as inconclusive with both techniques.

Contrary to our expectations, we found no difference in absolute prevalence of PE between the 64-slice and 4-slice CT scan cohorts. We did, however, observe a trend to more ISSPE among the 


\section{What is known about this topic?}

- With the introduction of multi-detector row computed tomography (CT), visualisation of small peripheral arteries has greatly improved, yielding an increased sensitivity for the detection of small subsegmental emboli.

- Newer generation CT scans may lead to a higher prevalence and a different distribution of pulmonary embolism (PE), with unknown clinical consequences.

\section{What does this paper add?}

- A comparison between 'low'-multi-detector row CT scans (i.e. 4-slice) and modern 'high-end'-multi-detector row CT scans (i.e. 64-slice) did not reveal a difference in absolute prevalence of $\mathrm{PE}$, although isolated subsegmental PE seemed higher with 64-slice CT.

- Careful evaluation, however, showed inconclusive tests results in a large proportion of patients, exceeding the number found in management studies.

- This may indicate that the diagnosis of PE with multidetector-row CT could be more difficult than assumed.

64-slice scans. The latter was expected and is explained by the better visualisation of small peripheral arteries. However, with an increase in the detection of (ISS-)PE, one would expect the absolute prevalence of PE to increase in this cohort as well; the proportion of central/segmental PE should not differ between the 4-and 64-slice cohort. This is most probably due to chance and overall caution must be taken interpreting the small patient groups. Another explanation could be that the indirect comparison resulted in differences between the two cohorts, although baseline characteristics and the hospital referral patterns were similar. Three radiologists evaluated every scan in both cohorts, making an explanation on the level of radiologists unlikely.

The prevalence of isolated subsegmental PE varies in the literature. Using MDCT, the prevalence ranged between $7.5 \%$ and $22 \%$ with 4-slice MDCT $(2,9)$, whereas this was $5.4 \%$ with 8-/16-slice MDCT (10). In addition, in studies that did not specify the type of MDCT, the prevalence was $14 \%$ and $18 \%(11,12)$. With re-evaluation of the scans by three experienced radiologists, we found a prevalence of $7.7 \%$ with 4 -slice MDCT and $21 \%$ for 64 -slice MDCT in our study, which corresponds to the range reported in the literature.

Currently, it is uncertain whether treating patients with ISSPE is beneficial, or would influence the three-month VTE rate after a 4or 64-slice CT scan $(10,13)$. In a retrospective study by Eyer et al. (10), there was no significant difference in outcome between patients with isolated subsegmental embolism who were and who were not treated with anticoagulants. However, more studies, preferably with a prospective randomised design, are necessary before this question can be answered with certainty.

Our second main finding refers to the high number of inconclusive CT-scans. Overall, scans were inconclusive in one in $10 \mathrm{pa}-$ tients, which is higher compared to the $1-2 \%$ observed in clinical management studies $(2,6,14)$. It has to be considered that evaluating scans in a research setting without management consequences is different from daily clinical practice. Also, a forced agreement between three radiologists inevitably causes more inconclusive outcomes than the evaluation by a single reader. Yet even when taking these two factors into account, our results suggest that in daily clinical practice treatment decisions might be based upon inconclusive CT scans in a subset of patients. The diagnosis of PE with MDCT could therefore be less straightforward than assumed and shows a considerable variability.

We found no difference in the total number of inconclusive scan results between the two cohorts. However, there seemed to be a trade-off in the reasons for the scan to be inconclusive. Because of the faster scanning time with 64-slice MDCT, it is not surprising that timing was a greater problem with these scans, while movement artefacts more often caused inconclusive outcome among the 4-slice CT scans. The inter-reader agreement between the three readers was substantial, although agreement between two readers (ranging from 0.62 to 0.80 ) was slightly lower compared to reports in the literature ranging from 0.84 to $0.97(15,16)$. There was no difference in inter-reader agreement between the 4- and 64-slice scanner.

Several aspects of our study require comment. First, this was a retrospective study comparing CT scans of patient from two separate cohorts. Although the baseline characteristics are comparable, it would have been preferable to compare the two MDCT types simultaneously in the same patient. However, this would require performing two CT scans in each patient, exposing them to a double radiation dose. Comparing CT scans from two similar sets of patients, as we have done, is the next best option.

Secondly, CT acquisition protocols differed with respect to slice geometry and contrast media application. This means that to a certain extent our results are valid only for these particular protocols and different protocols might yield slightly different results. On the other hand, both protocols represented state of the art procedures implemented in clinical routine.

Thirdly and most importantly, the number of patients included in this study was rather small and likely affected the power of our statistics. We were not able to prove a significant difference in ISSPE prevalence, which does not mean that there is none. In order to reach significance, 500 patients in each group should have been included. The 2.7-fold increase of ISSPE, however, suggests that more isolated peripheral emboli are detected with newer generation MDCT scans, while the clinical consequences of these findings are currently unknown (10).

In conclusion, although the absolute prevalence of PE was comparable in both cohorts, the prevalence of isolated subsegmental

\section{Abbreviations}

$\mathrm{Cl}$, confidence interval; $\mathrm{CT}$, computed tomography; CTPA, computed tomographic pulmonary angiography; ISSPE, isolated subsegmental pulmonary embolism; MDCT, multi detector-row computed tomography; PE, pulmonary embolism. 
PE seems higher with 64-slice CT scanning. Also, careful evaluation of the scans showed inconclusive test results in a larger proportion of patients compared with management studies, indicating that assessment of PE with MDCT could be more difficult than assumed.

\section{References}

1. Rathbun SW, Raskob GE, Whitsett TL. Sensitivity and specificity of helical computed tomography in the diagnosis of pulmonary embolism: a systematic review. Ann Intern Med 2000; 132: 227-232.

2. Perrier A, Roy PM, Sanchez O, et al. Multidetector-row computed tomography in suspected pulmonary embolism. N Engl J Med 2005; 352: 1760-1768.

3. Patel S, Kazerooni EA, Cascade PN. Pulmonary embolism: optimization of small pulmonary artery visualization at multi-detector row CT. Radiology 2003; 227: 455-460.

4. Ghaye B, Szapiro D, Mastora I, et al. Peripheral pulmonary arteries: how far in the lung does multi-detector row spiral CT allow analysis? Radiology 2001; 219: 629-636.

5. Wells PS, Anderson DR, Rodger M, et al. Derivation of a simple clinical model to categorize patients probability of pulmonary embolism: increasing the models utility with the SimpliRED D-dimer. Thromb Haemost 2000; 83: 416-420.

6. The Christopher study investigators, van Belle A, Buller HR, et al. Effectiveness of managing suspected pulmonary embolism using an algorithm combining clinical probability, D-dimer testing, and computed tomography. J Am Med Assoc 2006; 295: 172-179.
7. Boyden E. Segmental anatomy of the lungs. New York, NY: McGraw-Hill; 1955.

8. Jackson CL, Huber JF. Correlated applied anatomy of the bronchial tree and lungs with a system of nomenclature. Chest 1943; 9: 319-326.

9. Thomeer MG, Pattynama PM, Hartmann IJ, et al. High incidence of isolated subsegmental pulmonary emboli on multi-slice spiral CT: a comparative clinical study. Thromb Haemost 2006; 95: 914-915.

10. Eyer BA, Goodman LR, Washington L. Clinicians' response to radiologists' reports of isolated subsegmental pulmonary embolism or inconclusive interpretation of pulmonary embolism using MDCT. AJR Am J Roentgenol 2005; 184: 623-628.

11. Coche E, Verschuren F, Keyeux A, et al. Diagnosis of acute pulmonary embolism in outpatients: comparison of thin-collimation multi-detector row spiral CT and planar ventilation-perfusion scintigraphy. Radiology 2003; 229: 757-765.

12. Nijkeuter M, Kwakkel-van Erp JM, Kruip MJ, et al. Incidence of diagnosis of subsegmental pulmonary emboli using multidetector row and single-detector row computed tomography. J Thromb Haemost 2008; 6: 384-386.

13. Goodman LR. Small pulmonary emboli: what do we know? Radiology 2005; 234 : 654-658.

14. Righini M, Le Gal G, Aujesky D, et al. Diagnosis of pulmonary embolism by multidetector CT alone or combined with venous ultrasonography of the leg: a randomised non-inferiority trial. Lancet 2008; 371: 1343-1352.

15. Revel MP, Petrover D, Hernigou A, et al. Diagnosing pulmonary embolism with four-detector row helical CT: prospective evaluation of 216 outpatients and inpatients. Radiology 2005; 234: 265-273.

16. Brunot S, Corneloup O, Latrabe V, et al. Reproducibility of multi-detector spiral computed tomography in detection of sub-segmental acute pulmonary embolism. Eur Radiol 2005; 15: 2057-2063. 